\title{
Variabilité de l'efficacité curative d'inhibiteurs de la biosynthèse des stérols vis-à-vis de Venturia inaequalis
}

\author{
L Parisi 1, J Guillaumes 1, JM Olivier 2 \\ avec la collaboration technique de J Gaudin \\ ${ }^{1}$ INRA, Station de pathologie végétale, Beaucouzé, 49000 Angers; \\ 2 INRA, Station de recherches sur les champignons, BP 81, 33883 Villenave-d'Ornon Cedex, France
}

(Reçu le 28 décembre 1989; accepté le 18 juin 1990)

\begin{abstract}
Résumé - Des isolats de Venturia inaequalis, issus de vergers traités ou non par des inhibiteurs de la biosynthèse des stérols, présentent des différences de sensibilité in vitro vis-à-vis, notamment, de l'un d'entre eux : le fénarimol. Les concentrations inhibitrices les plus élevées sont observées pour les souches provenant de vergers traités. L'efficacité curative in vivo de 9 inhibiteurs de la biosynthèse des stérols vis-à-vis de 3 souches a été estimée par le pourcentage de plantes tavelées après contamination artificielle et traitement de plantules de pommier cultivées en chambre climatique. Une des souches issue d'un verger non traité est considérée comme sensible, alors que les 2 autres provenant de vergers traités sont considérées comme résistantes. Ainsi pour le fénarimol, la moitié de la dose autorisée est suffisante pour empêcher le développement de la souche sensible, tandis que la dose autorisée n'inhibe pas totalement les souches résistantes. Par ailleurs, les 2 souches résistantes, inhibées in vitro de façon semblable par le fénarimol, ont un comportement différent au niveau des plantules de pommier : l'une d'entre elles est mieux contrôlée que l'autre par les inhibiteurs de la biosynthèse des stérols. En outre, quelles que soient les souches, des différences d'activité curative s'observent entre les diverses formulations expérimentées. Les implications pratiques de ces résultats sont discutées.
\end{abstract}

tavelure / pommier / résistance aux fongicides / épidémiologie

Summary - Variability in the curative effects of sterol demethylation inhibitors towards Venturia inaequalis. Some strains of $\mathrm{V}$ inaequalis from orchards sprayed and unsprayed with sterol demethylation inhibitors (table I), showed in vitro variability in their sensitivity to fenarimol. Inhibiting concentrations were higher for the strains from sprayed orchards (table III and figure I). The in vivo efficacy of 9 sterol demethylation inhibitars (table II) towards 3 strains was estimated by the percentage of scabbed plants after artificial inoculation and treatment of plantlets cultivated in a growth chamber. One of the strains from an unsprayed orchard was considered as sensitive, while the 2 others from sprayed orchards were considered as resistant. As regards the use of fenarimol, half the normal rate was sufficient to control the sensitive strain, while the normal rate did not completely control the resistant strains (table IV). Otherwise, the 2 resistant strains, similarly inhibited in vitro by fenarimol, demonstrated different behaviour at the level of apple plantlets: one of them was better controlled by the sterol demethylation inhibitors than the other. Moreover, differences in the curative efficacy of the different compounds tested were observed for all the strains (tables $V$ and VI). Practical implications of these results are discussed.

apple orchard / scab / resistance to fungicides / epidemiology 


\section{INTRODUCTION}

Les fongicides inhibiteurs de la biosynthèse des stérols (IBS) sont actuellement largement employés dans la lutte contre la tavelure et l'oïdium du pommier. Ils offrent la possibilité de traitements curatifs contre Venturia inaequalis (Cke) Wint, de 3 à 5 j après la pluie contaminatrice, avec une bonne efficacité (Olivier, 1984; O'Leary et al, 1987).

Tous les IBS actuellement employés en arboriculture sont des inhibiteurs de la $\mathrm{C} 14$ déméthylation des stérols (fénarimol, nuarimol, bitertanol, flusilazol, triforine, pyrifenox, penconazole). Ils peuvent donc être considérés comme des fongicides unisites (Leroux, 1985). Cette caractéristique, ajoutée à un usage intensif, rend nécessaire une évaluation des risques de développement de souches résistantes.

Dans la pratique, des souches résistantes à ces IBS ont été détectées chez les agents des oïdiums des céréales et des cucurbitacées, du piétin-verse des céréales, de l'helminthosporiose et de la rhynchosporiose de l'orge (Leroux, $1987 \mathrm{a}$ et b).Pour la tavelure du pommier, il a été fait état de la présence de souches résistantes dans des vergers intensivement traités de différents pays européens (Stanis et Jones, 1985; Thind et al, 1986a et b; Fiaccadori et al, 1987). Aucune perte d'efficacité de la protection avec les IBS n'a été observée en Europe. Cependant, Hildebrand et al (1988) ont montré au Canada que l'usage intensif des IBS dans un verger expérimental avait entraîné une perte pratique d'efficacité des traitements, associée au développement d'une population résistante de $V$ inaequalis.

D'après les analyses réalisées sur des populations françaises de $V$ inaequalis, Morzières et al (1988) ont mis en évidence des souches "R" et «"», respectivement «fortement» et «moyennement» résistantes au fénarimol. Cette distinction est basée sur la croissance et l'aspect du tube germinatif de conidies déposées à la surface d'un milieu gélosé contenant $1 \mathrm{mg} / \mathrm{l}$ de fénarimol (Morzières et al, 1988).

$\mathrm{Si}$ la résistance de ces souches aux différentes matières actives a été étudiée in vitro (Thind et al, 1986a et b; Creemers et al, 1988) nous connaissons peu de choses sur leur réaction in vivo à des traitements curatifs effectués à dose homologuée. II nous a donc paru important d'étudier l'effet in vivo des divers IBS actuellement autorisés en france contre la tavelure du pommier, vis-à-vis de certaines de ces souches considérées comme résistant à ces fongicides.

Cette étude devrait permettre d'estimer le risque que peut réellement présenter la présence de ces souches dans les vergers.

\section{MATÉRIEL ET MÉTHODES}

\section{Souches de $\mathrm{V}$ inaequalis}

Les caractéristiques des différentes souches étudiées sont données par le tableau I.

\section{Croissance mycélienne sur milieu avec fongicide}

Les essais ont été conduits avec le fénarimol. Le fongicide (spécialité commerciale : Rubigan 4) est ajouté à du malt gélosé (Cristomalt de Difal à $1 \%$ ) maintenu en surfusion. Huit concentrations de matière active ont été testées : 0,$1 ; 0,25 ; 0,5 ; 1 ; 2,5 ; 5 ; 7,5$ et $10 \mathrm{mg} / \mathrm{l}$. Par souche et par concentration, 5 boîtes de Petri sont ensemencées avec 4 implants de $4 \mathrm{~mm}$ de diamètre pris à la périphérie d'une colonie de $V$ inaequalis âgée d'environ 1 mois. La croissance se fait à l'obscurité à une température de $16 \pm 1^{\circ} \mathrm{C}$ pendant 1 mois, puis le diamètre de chaque colonie est mesuré.

Le pourcentage d'inhibition de la croissance mycélienne en présence de fongicide est calculé comparativement aux témoins sans fongicide, puis les concentrations inhibant $50 \%$ et $90 \%$ de la croissance mycélienne ( $\mathrm{Cl} 50$ et 90 ) sont estimées graphiquement.

\section{Efficacité des traitements curatifs sur plantules de pommier inoculées par $\mathrm{V}$ inaequalis}

La méthode employée est celle décrite par Olivier et Lespinasse (1980), avec une modification : les plantules de pommier sont issues de pépins provenant d'un croisement entre les deux variétés Golden Delicious et Granny Smith, sensibles à la tavelure. Elles sont contaminées quand elles ont atteint le stade 3 feuilles.

Les suspensions conidiennes, préparées selon la méthode de Keitt et Palmiter (1938) et ajustées à une concentration voisine de $3.10^{5}$ conidies $/ \mathrm{ml}$, sont pulvérisées à raison de $25 \mathrm{ml}$ de suspension par lot de 34 plantules, effectif moyen pour chaque essai (soit 3 répétitions de 11 à 12 plantules chacune). Une humidité saturante est maintenue pendant les premiers stades de l'infection, en plaçant les plantules dans une enceinte plastique qui est retirée $48 \mathrm{~h}$ après 
Tableau I. Souches de $V$ inaequalis étudiées.

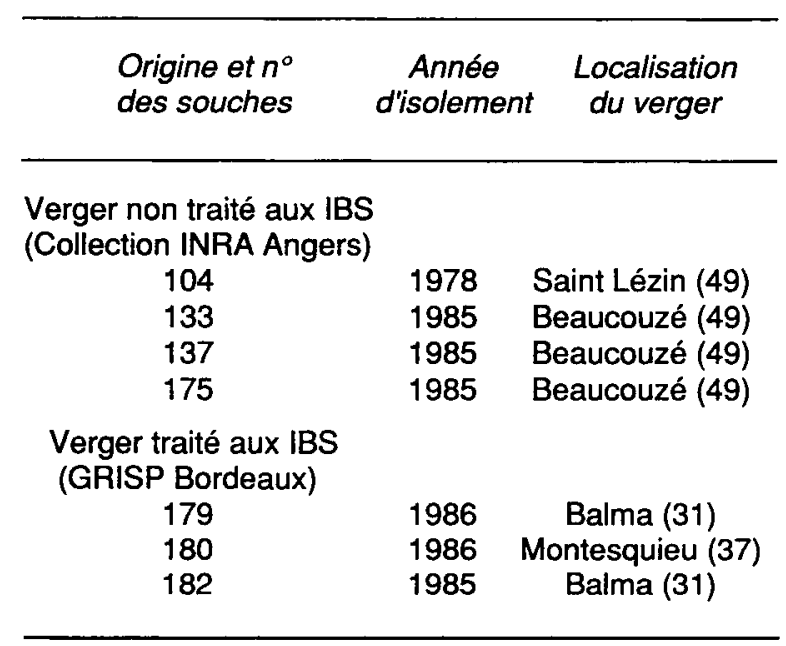

l'inoculation. Les traitements fongicides sont réalisés 48 à $67 \mathrm{~h}$ après contamination avec un pulvérisateur manuel, à raison de $20 \mathrm{ml}$ de fongicide par lot de 34 plantes.

Les plantes sont ensuite élevées entre 18 et $20^{\circ} \mathrm{C}$ avec une humidité relative de 70 à $90 \%$ et un éclairage de $16 \mathrm{~h}$ par jour (3 000 lux au niveau des feuilles).
Les produits expérimentés ainsi que les doses utilisées (correspondant à celles autorisées en pratique) sont mentionnés dans le tableau II. Le fénarimol a également été essayé aux doses de 10,20 et $30 \mathrm{mg} /$ I, inférieures à celle autorisée en France qui est de 40 $\mathrm{mg} / \mathrm{l}$.

Deux ou trois notations ont été effectuées au cours du temps, suivant l'évolution de la maladie. Le pourcentage de plantes tavelées a été calculé dans chaque cas. Après transformation des pourcentages en valeurs arc sinus, les données ont été traitées par analyse de variance. Quand la valeur de F était significative au seuil $5 \%$, les moyennes pour chaque souche (tous fongicides confondus) ont été comparées par le test de Duncan.

\section{RÉSULTATS}

\section{Effet du fénarimol sur la croissance mycélienne}

Cet essai montre (tableau III) une grande variabilité dans la réponse des divers isolats de $V$ inaequalis vis-à-vis du fénarimol. Ainsi, pour les

Tableau II. Inhibiteurs de la biosynthèse des stérols expérimentés et doses d'utilisation.

* CE : concentré émulsionnable; PM : poudre mouillable; GS : granulés solubles dans l'eau.

\begin{tabular}{|c|c|c|c|c|}
\hline $\begin{array}{c}\text { Spécialité } \\
\text { commerciale }\end{array}$ & Firme & Formulation* & Composition & $\begin{array}{c}\text { Doses autorisées : } \\
m g / l \text { de matières actives }\end{array}$ \\
\hline Rubigan 4 & Lilly & $\mathrm{CE}$ & Fénarimol $40 \mathrm{~g} / \mathrm{l}$ & 40 \\
\hline Baycor 25 & Bayer & PM & Bitertanol $25 \%$ & 187,5 \\
\hline Nustar 40 EC & Du Pont de Nemours & CE & Flusilazol $400 \mathrm{~g} / \mathrm{l}$ & 20 \\
\hline Saprol & Prochimagro & $\mathrm{CE}$ & Triforine $190 \mathrm{~g} / \mathrm{l}$ & 380 \\
\hline Erisan Super & Pepro & PM & $\begin{array}{c}\text { Fenarimol 1\% } \\
+ \\
\begin{array}{c}+ \\
\text { Mancozèbe } 40 \% \\
+ \\
\text { Captane } 25 \%\end{array}\end{array}$ & $\begin{array}{c}20 \\
+ \\
800 \\
+ \\
500\end{array}$ \\
\hline Prevalan & Schering & GS & $\begin{array}{c}\text { Nuarimol } 2,4 \% \\
+ \\
\text { Captane } 72,6 \%\end{array}$ & $\begin{array}{c}24 \\
+ \\
726\end{array}$ \\
\hline Spontal & Sandoz & PM & $\begin{array}{c}\text { Nuarimol } 1 \% \\
+ \\
\text { Macozèbe } 60 \%\end{array}$ & $\begin{array}{c}20 \\
+ \\
1200\end{array}$ \\
\hline Topaze C & Ciba-Geigy & PM & $\begin{array}{c}\text { Penconazole } 2,5 \% \\
+ \\
\text { Captane } 47,5 \%\end{array}$ & $\begin{array}{c}25 \\
+ \\
475\end{array}$ \\
\hline Rondo & La Quinoleine & PM & $\begin{array}{c}\text { Pyrifénox 5\% } \\
+ \\
\text { Captane } 60 \%\end{array}$ & $\begin{array}{c}50 \\
+ \\
600\end{array}$ \\
\hline
\end{tabular}


souches issues de vergers non traités aux IBS, les $\mathrm{Cl} 50$ sont comprises entre 0,15 et $0,85 \mathrm{mg} / \mathrm{l}$ et leurs $\mathrm{Cl} 90$ entre 1,0 et $2,35 \mathrm{mg} / \mathrm{l}$. Quant à celles provenant de vergers traités, elles apparaissent moins sensibles au fénarimol puisque les $\mathrm{Cl} 50$ sont comprises en 1,90 et $2,60 \mathrm{mg} / \mathrm{l}$ et les $\mathrm{Cl} 90$ entre 4,90 et $10,00 \mathrm{mg} / \mathrm{l}$.

La figure 1 donne la courbe de réponse au fénarimol in vitro pour les 3 souches 104, 179 et 180. La croissance mycélienne de la souche 179 n'est pas totalement inhibée par $10 \mathrm{mg} / \mathrm{l}$ de fénarimol, tandis que la souche 104 est inhibée dès $2,5 \mathrm{mg} / \mathrm{l}$. La réponse de la souche 180 paraît intermédiaire entre celles des souches 104 et 179.

\section{Efficacité des divers IBS en traitements curatifs}

Parmi les souches provenant de vergers traités aux IBS, seules les souches 179 et 180 ont été testées au cours de ces expériences car la souche 182 n'a pas sporulé en culture pure.

Le tableau IV détaille les résultats de l'essai au cours duquel le fénarimol a été appliqué $55 \mathrm{~h}$ après inoculation, à des concentrations égales ou inférieures à celle qui est autorisée. L'efficacité du fongicide sur la souche 104 est complète dès la dose de $20 \mathrm{mg} /$, soit la moitié de la dose homologuée; quant à la dose de 10 $\mathrm{mg} / \mathrm{l}$, elle permet de limiter fortement l'expression de la maladie $(5,8 \%$ de plantes tavelées).

Par contre, la sensibilité au fénarimol des 2 souches 179 et 180 est plus faible que celle de la 104 : aucune dose n'aboutit à une bonne efficacité, même la dose homologuée. Toutefois le pourcentage de plantules malades diminue quand la dose de fénarimol augmente.

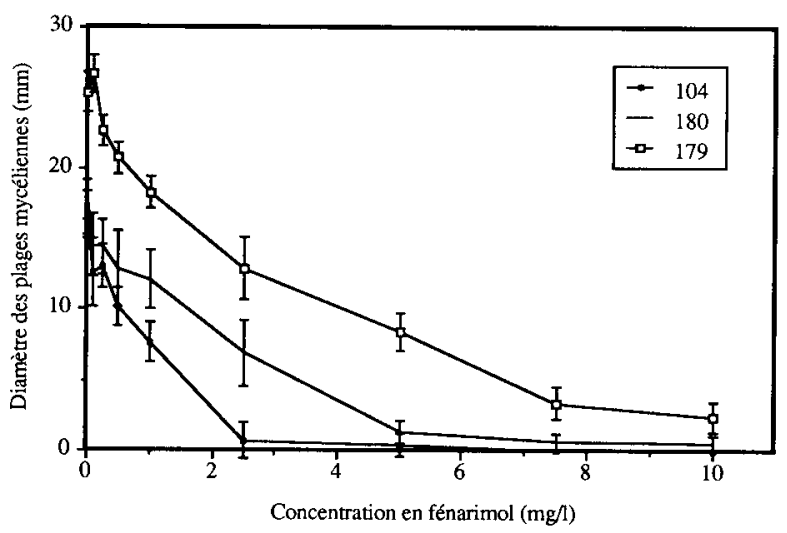

Fig 1. Inhibition in vitro de la croissance mycélienne de 3 souches de $V$ inaequalis par le fénarimol.
Tableau III. Concentrations de fénarimol inhibant 50 et $90 \%$ de la croissance mycélienne de $V$ inaequalis.

\begin{tabular}{ccc}
\hline & \multicolumn{3}{c}{ Concentration fénarimol $(\mathrm{mg} / \mathrm{l})$} \\
& $\mathrm{Cl} 50$ & $\mathrm{Cl} 90$ \\
& & \\
\hline & & \\
133 & 0,15 & 1,00 \\
175 & 0,30 & 2,35 \\
137 & 0,60 & 1,50 \\
104 & 0,85 & 2,30 \\
180 & 1,90 & 4,90 \\
182 & 2,50 & 6,70 \\
179 & 2,60 & 10,00 \\
\hline
\end{tabular}

L'activité de 9 IBS (seuls ou associés avec un fongicide multi-site), utilisés curativement $48 \mathrm{~h}$ après inoculation, c'est-à-dire largement avant leurs délais maximaux d'utilisation qui sont de 4 à $5 \mathrm{j}$, est reportée dans le tableau $\mathrm{V}$.

Les témoins non traités montrent que les conditions de l'essai sont favorables à la tavelure.

L'inhibition de la souche 104 est totale, 14 j après l'inoculation, pour 7 des 9 fongicides testés. Par la suite, la protection demeure complète avec le flusilazol et le fénarimol, alors qu'un

Tableau IV. Effet du fénarimol à différentes doses, appliqué curativement $55 \mathrm{~h}$ après inoculation de 3 souches de $V$ inaequalis sur plantules de pommier. $a, b$ : les souches suivies de différentes lettres alphabétiques diffèrent significativement par le test de Duncan au seuil $5 \%$.

Souches

$104 a 179 b \quad 180 b$ $\begin{array}{ccc}\text { Dose de } & \text { Nombre } & \\ \text { fénarimol } & \text { de jours après } & \text { Pourcentage de } \\ \text { contamination } & \text { plantes tavelées }\end{array}$

\begin{tabular}{lrrrr}
\hline \multirow{2}{*}{ Sans traitement } & $12 \mathrm{j}$ & 100 & 91,2 & 76,5 \\
& $16 \mathrm{j}$ & 100 & 95,6 & 79,4 \\
$10 \mathrm{mg} / \mathrm{l}$ & $12 \mathrm{j}$ & 5,8 & 48,5 & 42,8 \\
& $16 \mathrm{j}$ & 5,8 & 72,7 & 62,8 \\
$20 \mathrm{mg} / \mathrm{l}$ & $12 \mathrm{j}$ & 0 & 21,2 & 63,6 \\
& $16 \mathrm{j}$ & 0 & 27,2 & 63,6 \\
$30 \mathrm{mg} / \mathrm{l}$ & $12 \mathrm{j}$ & 0 & 18,2 & 21,2 \\
& $16 \mathrm{j}$ & 0 & 30,3 & 36,4 \\
$40 \mathrm{mg} / /$ & $12 \mathrm{j}$ & 0 & 18,2 & 15,1 \\
& $16 \mathrm{j}$ & 0 & 24,2 & 36,4 \\
\hline
\end{tabular}


Tableau V. Effets de 9 inhibiteurs de la biosynthèse des stérols, seuls ou en association, appliqués curativement $48 \mathrm{~h}$ après inoculation de 3 souches de $V$ inaequalis sur plantules de pommier.

a, b, c : les souches suivies de différentes lettres alphabétiques diffèrent significativement par le test de Duncan au seuil $5 \%$.

\begin{tabular}{|c|c|c|c|c|}
\hline \multirow{2}{*}{$\begin{array}{l}\text { Nature du } \\
\text { traitement }\end{array}$} & \multirow{2}{*}{$\begin{array}{c}\begin{array}{c}\text { Nombre } \\
\text { de jours après } \\
\text { contamination }\end{array} \\
\\
14 \mathrm{j} \\
23 \mathrm{j}\end{array}$} & $\begin{array}{c}104 a \\
\text { Pou } \\
\text { plar }\end{array}$ & $\begin{array}{l}\text { ouches } \\
179 \mathrm{~b} \\
\text { centag } \\
\text { es tave }\end{array}$ & dées \\
\hline & & $\begin{array}{l}61,6 \\
94,4\end{array}$ & $\begin{array}{l}96,9 \\
100\end{array}$ & $\begin{array}{l}55,8 \\
61,7\end{array}$ \\
\hline Fénarimol & $\begin{array}{l}14 j \\
23 j\end{array}$ & $\begin{array}{l}0 \\
0\end{array}$ & $\begin{array}{c}0 \\
9,1\end{array}$ & $\begin{array}{r}9,4 \\
31,2\end{array}$ \\
\hline Bitertanol & $\begin{array}{l}14 j \\
23 j\end{array}$ & $\begin{array}{l}0 \\
6,2\end{array}$ & $\begin{array}{c}0 \\
17,6\end{array}$ & $\begin{array}{l}56,2 \\
65,6\end{array}$ \\
\hline Flusilazol & $\begin{array}{l}14 j \\
23 j\end{array}$ & $\begin{array}{l}0 \\
0\end{array}$ & $\begin{array}{c}0 \\
5,4\end{array}$ & $\begin{array}{r}6,2 \\
28,1\end{array}$ \\
\hline Triforine & $\begin{array}{l}14 j \\
23 j\end{array}$ & $\begin{array}{c}0 \\
17,6\end{array}$ & $\begin{array}{l}2,7 \\
54\end{array}$ & $\begin{array}{l}50 \\
58,8\end{array}$ \\
\hline $\begin{array}{l}\text { Fénarimol + } \\
\text { Mancozèbe + } \\
\text { Captane }\end{array}$ & $\begin{array}{l}14 j \\
23 j\end{array}$ & $\begin{array}{l}0 \\
2,9\end{array}$ & $\begin{array}{r}9,1 \\
21,2\end{array}$ & $\begin{array}{l}55,9 \\
61,8\end{array}$ \\
\hline $\begin{array}{l}\text { Nuarimol + } \\
\text { Captane }\end{array}$ & $\begin{array}{l}14 j \\
23 j\end{array}$ & $\begin{array}{l}12,5 \\
34,3\end{array}$ & $\begin{array}{l}29 \\
90,3\end{array}$ & $\begin{array}{l}39,4 \\
42,4\end{array}$ \\
\hline $\begin{array}{l}\text { Nuarimol + } \\
\text { Mancozèbe }\end{array}$ & $\begin{array}{l}14 j \\
23 j\end{array}$ & $\begin{array}{l}0 \\
2,8\end{array}$ & $\begin{array}{l}2,7 \\
25\end{array}$ & $\begin{array}{l}37,8 \\
51,3\end{array}$ \\
\hline $\begin{array}{l}\text { Penconazole + } \\
\text { Captane }\end{array}$ & $\begin{array}{l}14 j \\
23 j\end{array}$ & $\begin{array}{c}0 \\
10,8\end{array}$ & $\begin{array}{c}0 \\
16,2\end{array}$ & $\begin{array}{l}21 \\
34,2\end{array}$ \\
\hline $\begin{array}{l}\text { Pyrifénox + } \\
\text { Captane }\end{array}$ & $\begin{array}{l}14 j \\
23 j\end{array}$ & $\begin{array}{l}3 \\
6\end{array}$ & $\begin{array}{c}0 \\
19,3\end{array}$ & $\begin{array}{r}5,9 \\
44,1\end{array}$ \\
\hline
\end{tabular}

pourcentage faible de plantes tavelées est observé avec les 5 autres fongicides : bitertanol, triforine, fénarimol + mancozèbe +captane, nuari$\mathrm{mol}+$ mancozèbe, penconazole + captane. Avec les associations nuarimol + captane et pyrifenox + captane, la tavelure est décelée dès $14 \mathrm{j}$ après l'inoculation, mais seule une nette progression s'observe avec la $1^{\text {re }}$ association.

Dans cet essai, on remarque la différence, statistiquement significative, entre les 2 souches résistantes aux IBS : la souche 179 est mieux contrôlée que la souche 180 , alors qu'elle paraît moins sensible au fénarimol in vitro (tableau III). Pour cette dernière, aucun fongicide ne parvient à éviter le développement de symptômes $14 \mathrm{j}$ après l'inoculation, les plus efficaces étant le flusilazol, le fénarimol et l'association pyrifénox + captane; à la $2^{\mathbf{e}}$ notation, la maladie progresse pour tous les traitements, et seuls le flusilazol et l'association pyrifénox + captane ont une efficacité proche de $50 \%$.

Pour la souche 179, l'efficacité apparente est parfaite pour 5 des 9 fongicides à la première notation (fénarimol, bitertanol, flusilazol, penconazole + captane et pyrifénox + captane). Cependant, la $2^{e}$ notation montre, pour tous les traitements, des symptômes de tavelure. Dans cet essai, on note une différence d'efficacité entre les 2 associations contenant du nuarimol.

Le tableau VI détaille les résultats de l'essai d'efficacité de 8 IBS (seuls ou associés avec un

Tableau VI. Effets de 8 inhibiteurs de la biosynthèse des stérols, seuls ou en association, appliqués curativement $67 \mathrm{~h}$ après inoculation de 2 souches de $V$ inaequalis sur plantules de pommier.

\begin{tabular}{|c|c|c|c|}
\hline \multirow{2}{*}{$\begin{array}{c}\begin{array}{c}\text { Nature du } \\
\text { traitement }\end{array} \\
\text { Sans traitement }\end{array}$} & \multirow{2}{*}{$\begin{array}{c}\begin{array}{c}\text { Nombre } \\
\text { de jours après } \\
\text { contamination }\end{array} \\
\begin{array}{c}15 j \\
18 j\end{array}\end{array}$} & \multicolumn{2}{|c|}{$\begin{array}{l}\text { Souches } \\
104 \mathrm{a} 179 \mathrm{~b} \\
\text { Pourcentage de } \\
\text { plantes tavelées }\end{array}$} \\
\hline & & $\begin{array}{l}88,7 \\
96,2\end{array}$ & $\begin{array}{l}88,8 \\
96,3\end{array}$ \\
\hline Fénarimol & $\begin{array}{l}15 j \\
18 j \\
25 j\end{array}$ & $\begin{array}{l}0 \\
0 \\
2,8\end{array}$ & $\begin{array}{c}0 \\
0 \\
15,6\end{array}$ \\
\hline Flusilazol & $\begin{array}{l}15 j \\
18 j \\
25 j\end{array}$ & $\begin{array}{l}0 \\
0 \\
0\end{array}$ & $\begin{array}{l}0 \\
0 \\
5,7\end{array}$ \\
\hline Triforine & $\begin{array}{l}15 j \\
18 j \\
25 j\end{array}$ & $\begin{array}{l}0 \\
0 \\
0\end{array}$ & $\begin{array}{l}42,4 \\
48,5 \\
66,6\end{array}$ \\
\hline $\begin{array}{l}\text { Fénarimol + } \\
\text { Mancozèbe + } \\
\text { Captane }\end{array}$ & $\begin{array}{l}15 j \\
18 j \\
25 j\end{array}$ & $\begin{array}{c}9 \\
11,4 \\
34,3\end{array}$ & $\begin{array}{l}25 \\
38,8 \\
63,8\end{array}$ \\
\hline $\begin{array}{l}\text { Nuarimol + } \\
\text { Captane }\end{array}$ & $\begin{array}{l}15 j \\
18 j \\
25 j\end{array}$ & $\begin{array}{l}75 \\
78,1 \\
81,2\end{array}$ & $\begin{array}{l}29,4 \\
38,2 \\
54,5\end{array}$ \\
\hline $\begin{array}{l}\text { Nuarimol + } \\
\text { Mancozèbe }\end{array}$ & $\begin{array}{l}15 j \\
18 j \\
25 j\end{array}$ & $\begin{array}{r}6,4 \\
12,9 \\
12,9\end{array}$ & $\begin{array}{l}11,8 \\
26,5 \\
55,9\end{array}$ \\
\hline $\begin{array}{l}\text { Penconazole + } \\
\text { Captane }\end{array}$ & $\begin{array}{l}15 j \\
18 j \\
25 j\end{array}$ & $\begin{array}{l}0 \\
0 \\
0\end{array}$ & $\begin{array}{l}0 \\
0 \\
0\end{array}$ \\
\hline $\begin{array}{l}\text { Pyrifénox + } \\
\text { Captane }\end{array}$ & $\begin{array}{l}15 j \\
18 j \\
25 j\end{array}$ & $\begin{array}{l}0 \\
0 \\
0\end{array}$ & $\begin{array}{l}0 \\
0 \\
0\end{array}$ \\
\hline
\end{tabular}


fongicide multi-site) utilisés curativement $67 \mathrm{~h}$ après inoculation des souches 104 et 179 . Les conditions expérimentales sont donc sévères pour un produit comme la triforine, dont le délai d'utilisation en curatif est de $72 \mathrm{~h}$ après inoculation. Pour les autres fongicides étudiés, le délai recommandé pour l'utilisation en curatif est de $96 \mathrm{~h}$, sauf pour le flusilazol et l'association pyrifénox + captane, où il est de $120 \mathrm{~h}$.

Vis-à-vis de la souche 104, 5 fongicides ont une bonne efficacité; ce sont le fénarimol, le flusilazol, la triforine, les associations penconazole et périfénox + captane. Par contre, avec les associations comportant du nuarimol et celle à base de fénarimol, des symptômes sont visibles; dans le dernier cas il faut noter que le fénarimol est apporté à la dose de $20 \mathrm{mg} / \mathrm{l}$, alors qu'utilisé seul il est recommandé à la dose de $40 \mathrm{mg} / \mathrm{l}$.

Les résultats avec la souche 179 montrent une bonne efficacité des associations du penconazole et du pyrifénox avec le captane, et une assez bonne efficacité du flusilazol et du fénarimol. Par contre, le pourcentage de feuilles tavelées est plus élevé par rapport à la souche sensible, pour les associations nuarimol + mancozèbe et fénarimol + mancozèbe + captane $(63,8 \%)$, et plus faible pour le nuarimol + captane. C'est avec la triforine, pulvérisée à la limite de son délai d'utilisation, que la différence est la plus grande entre les 2 souches : $42,4 \%$ de plantes malades, puis $66,6 \%$ à la dernière notation, alors que l'efficacité est totale pour la souche 104.

\section{DISCUSSION}

Les souches 179,180 et 182 issues de vergers ayant reçu de nombreux traitements à base d'IBS ont des caractéristiques similaires à celles décrites par Stanis et Jones (1985), Fiaccadori et al (1987) et Creemers et al (1988), et considérées comme résistantes à ces fongicides.

Deux des souches étudiées sont issues du verger de Balma; au moment de leur isolement, elles ont été caractérisées comme "R", soit fortement résistantes (Thind et al, 1986a). Les tests réalisés sur spores simultanément aux essais rapportés dans le présent article les placent plutôt dans le type "r", avec un facteur de résistance plus faible. Cette observation pourrait suggérer que le caractère de forte résistance serait instable. Des essais complémentaires devront le vérifier; il faut cependant prendre en compte les difficultés rencontrées dans le calcui des fac- teurs de résistance, liées à la forte variabilité des souches de $V$ inaequalis considérées comme sensibles.

Peu de choses étaient connues concernant le contrôle in vivo des souches résistantes par les différents IBS actuellement homologués pour la lutte contre la tavelure du pommier. Seuls Thind et al (1986a) ont étudié en serre l'action du fénarimol sur une souche résistante et mis en évidence la mauvaise efficacité de traitements curatifs effectués $3 \mathrm{j}$ après la contamination.

Notre travail montre que les souches 179 et 180 se différencient significativement de la souche 104, isolée dans un verger non traité aux IBS et considérée comme sensible. Ce résultat ne peut être expliqué par une différence d'agressivité, celle de la souche 180 n'étant que légèrement inférieure à celle des souches 179 et 104.

Une première expérience montre que l'inhibition des 2 souches résistantes est fonction de la dose de fénarimol employée et n'est jamais complète, contrairement à la souche sensible, totalement inhibée par un traitement à demi-dose. L'une des souches résistantes (180) est mal contrôlée par tous les IBS testés, utilisés en curatif. Pour la souche 179 , l'efficacité des traitements est mauvaise en situation «difficile" : délai d'utilisation en curatif proche du maximum autorisé, ou formulations apportant une dose réduite d'IBS.

Par ailleurs, la différence entre nuarimol + captane et nuarimol + mancozèbe doit être mentionnée, car pour les 3 souches testées l'efficacité curative est meilleure avec l'association au dosage en IBS le plus faible. Nous ignorons si ce résultat est lié à la formulation ou à la nature du produit de contact associé avec l'IBS.

Ces résultats montrent que la présence de souches résistantes dans un verger est susceptible d'entraîner une perte d'efficacité des traitements avec les IBS seuls ou associés avec des fongicides multi-sites; ce risque doit être pris en compte. Ils sont en accord avec ceux obtenus au verger par Hildebrand et al (1988). Ces auteurs observent dans un verger intensivement traité aux IBS (7 à 9 traitements par an pendant 10 ans) une perte pratique d'efficacité, par paliers, aboutissant à une inefficacité totale des traitements la dernière année. Les isolats de $V$ inaequalis les plus résistants issus de ce verger ont des facteurs de résistance de 22 vis-à-vis du bitertanol, IBS le plus employé dans cette expérimentation. 
Cependant, nous ignorons actuellement quel est le seuil de souches résistantes au-delà duquel on risque de voir apparaître des échecs de traitements. L'acquisition de données complémentaires concernant la valeur adaptative des souches résistantes et leur persistance dans les vergers après l'arrêt des traitements paraît indispensable.

Les IBS, seuls ou associés à des fongicides multi-sites, sont actuellement de plus en plus employés dans la lutte contre la tavelure et l'oïdium du pommier. Leur intérêt réside surtout dans leur pouvoir curatif, permettant d'intervenir jusqu'à $5 \mathrm{j}$ après le début de la pluie contaminatrice. Par contre, leur durée d'action préventive, de 3 à $4 \mathrm{j}$, est plus faible que celle des fongicides multi-sites de contact.

II paraît nécessaire, pour éviter l'émergence d'une résistance en pratique et préserver leur efficacité, de raisonner les modalités d'application des IBS dans les vergers. Une stratégie devra être élaborée pour une utilisation optimale de ces fongicides. En pratique, notre travail apporte une contribution à cette réflexion. En effet, il paraît souhaitable de ne pas utiliser les IBS curativement, seuls ou associés à un fongicide multi-site, à une dose inférieure à la dose homologuée. Dans ce cas, si leur efficacité reste excellente en présence de souches sensibles, elle est insuffisante en présence de souches résistantes; l'utilisation à pleine dose est donc préférable.

\section{REMERCIEMENTS}

Les auteurs remercient M Clerjeau (INRA Bordeaux) pour la fourniture des souches résistantes, et $Y$ Lespinasse (INRA Angers) pour celle du matériel végétal nécessaire aux essais, ainsi que $M$ Lefeuvre (CETA Anjou) et G Lachaud (INRA Angers) pour leur aide.

\section{RÉFÉRENCES}

Creemers P, Vandergeten J, Vanmechelen A (1988) Variability in sensitivity of fields isolates of Venturia sp to demethylation inhibitors. Med Fac Landbouww Rijksuniv Gent 53/2b, 577-587

Fiaccadori R, Gienlink AJ, Dekker J (1987) Sensitivity to inhibitors of sterol biosynthesis in isolates of Venturia inaequalis from Italian and Deutch orchards. Neth J Plant Patho/ 93, 285-287

Hildebrand PD, Lockhart CL, Newbery RJ, Ross RG (1988) Resistance of Venturia inaequalis to bitertanol and other demethylation-inhibiting fungicides. Can J Plant Pathol 10, 311-316

Keitt GW, Palmiter DH (1938) Heterothallism and variability in Venturia inaequalis. Am J Bot 25, 338-345

Leroux $P$ (1985) Les phénomènes de résistance des champignons phytopathogènes aux fongicides. Maladies des plantes : Journées d'étude ANPP 1, $19-45$

Leroux $P$ (1987a) La résistance des champignons aux fongicides. 1-Phytoma 385, 6-14

Leroux $P$ (1987b) La résistance des champignons aux fongicides. 2-Phytoma 386, 31-35

Morzières JP, Clerjeau M, Thind TS (1988) Mise au point sur la résistance des souches de Venturia inaequalis aux fongicides inhibiteurs de la biosynthèse de l'ergostérol en France. Maladies des plantes : Journées d'études ANPP 2, 1321-1326

O'Leary AL, Jones AL, Ehret GR (1987) Application rates and spray intervals for apple scab control with flusilazol and pyrifenox. Plant Dis 71, 623-626

Olivier JM (1984) Évolution de la lutte contre la tavelure du pommier. Déf Vég 225, 22-35

Olivier JM, Lespinasse $Y$ (1980) Étude de l'efficacité de fongicides antitavelures après contamination de pommiers en serre. 1-Méthode d'étude sur jeunes semis. Phytiatr Phytopharm 29, 13-22

Stanis VF, Jones AL (1985) Reduced sensitivity to sterol inhibiting fungicides in field isolates of Venturia inaequalis. Phytopathology 75, 1098-1101

Thind T, Clerjeau M, Olivier JM (1986a) First observations on resistance in Venturia inaequalis and Guinardia bidwellii to ergosterol biosynthesis inhibitors in France. Proc British Protec Conf Pests Dis 2, 491-498

Thind T, Olivier JM, Clerjeau M (1986b) Tavelure du pommier : mise en évidence d'une résistance aux fongicides inhibiteurs de la biosynthèse de l'ergostérol. Phytoma 381, 13-16 